\title{
With HEART Towards Response Time Guarantees for Message-Based e-Services
}

\author{
Achim Kraiss ${ }^{2 *}$, Frank Schoen ${ }^{1}$, Gerhard Weikum ${ }^{3}$, and Uwe Deppisch ${ }^{1}$ \\ ${ }^{1}$ Dresdner Bank, Software-Technology and -Architecture, IT Research and Innovations \\ \{frank.schoen, uwe.deppisch\}@dresdner-bank.com \\ ${ }^{2}$ SAP AG, Customer Relationship Management \\ achim.kraissesap.com \\ ${ }^{3}$ University of the Saarland, Computer Science Department \\ weikumecs. uni-sb. de
}

\begin{abstract}
The HEART tool (Help for Ensuring Acceptable Response Times) has been developed by the IT Research and Innovations department of Dresdner Bank for the computation of viable message prioritization in message-based eservices, such as stock brokerage services where service requests of different customer classes with class-specific performance goals have to be served by a server. HEART determines viable message prioritizations in the sense that they satisfy the specified performance goals of customer classes. In this paper, we describe the practical problem setting we address with HEART and outline the functionality of HEART. The demo will show HEART's underlying concepts, its architecture and an example scenario.
\end{abstract}

\section{Motivation}

Quality-of-Service (QoS) is a hot topic for existing Internet-based e-commerce applications and it will become "hotter" as enterprises make more and more Web Services accessible to a wider range of customers via Internet, using UDDI registries and SOAP-based messaging. Guaranteeing good performance is highly critical to the acceptance and business success of e-services [1], such as online stock brokerage where minimum response times are required by customers for their trading transactions such as buying and selling of stocks. In this setting, it is not sufficient to the customer to get "best-effort" promises about the e-service performance, but to get specific performance guarantees which may even be cast into a formal service level agreement (SLA). These performance guarantees have not only to focus on mean response times averaged over long time periods like weeks or months. Rather they have to consider mean values, standard deviations as well as the tail of response time distributions taken in short-term intervals in order to guarantee acceptable response times even in peak load situations where the number of response time outliers may be most critical to business success. Consequently, future e-service servers have to guarantee customer-class-specific performance goals. Typically, e-services are embedded in a component-based architecture where requests (e.g. in form of a SOAP message or

\footnotetext{
* This work was performed while the author was at Dresdner Bank.
} 
as a message within some message-oriented middleware (MOM)) are sent to the eservice server where the request is processed and the answer is sent back to the service requestor. If the server serves all the incoming requests in first-come-first-served (FCFS) order, it is not possible to guarantee customer-class-specific response times. A commonly provided base mechanism to guarantee some form of class-specific response times is to assign different priorities to requests of different customer classes and to serve incoming requests based on their priorities. Many MOM products, such as IBM's MQSeries and Microsoft's MSMQ, as well as messaging interfaces such as Java Message Service (JMS) provide message priorities and priority-based message scheduling as basis for influencing message-class specific response times. However, in practice it is completely unclear how to set message priorities in order to satisfy given response time goals, and this is why we have developed the HEART tool (Help for Ensuring Acceptable Response Times).

\section{Example Problem Setting}

Figure 1 illustrates the problem setting we address with HEART. A server provides stock brokerage services, such as for the buying of stocks (BuyStocks), and for querying the current portfolio of the customer (QueryPortfolio). These services are accessed by three different customer classes: (1) first-class customers who pay for their brokerage services (e.g. stock brokers), (2) second-class customers who can use the brokerage for free (e.g. a service for students), and (3) inhouse applications (e.g. for recording written customer orders). The first-class and second-class customer applications may send SOAP messages with their service requests over the Internet to a web server of the bank where the service requests are transferred into the request queue of the brokerage server using message-oriented middleware. The inhouse applications may directly send MOM messages to the request queue. Each customer class has specific performance goals for their service requests. The first-class customers require

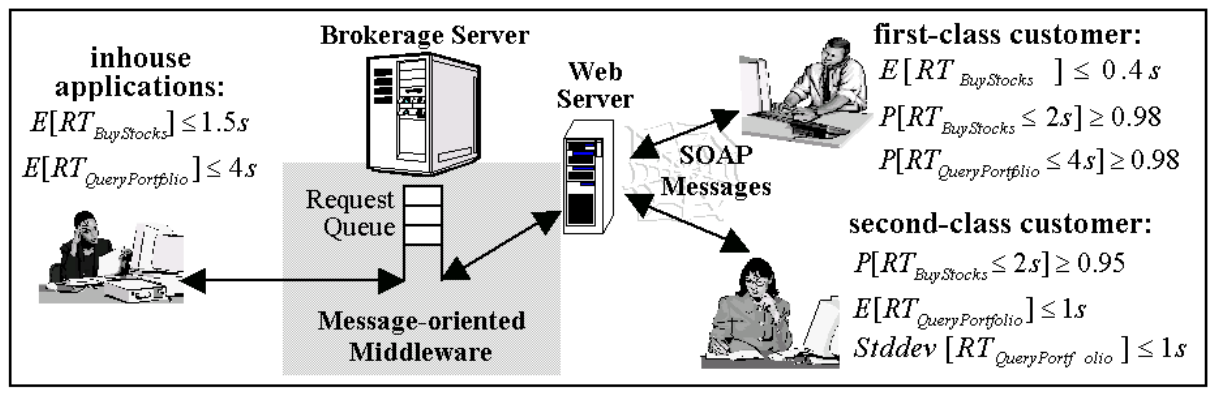

Fig. 1. Example scenario for message-based e-services

that the mean response time of a BuyStocks request does not exceed 0.4 seconds. Furthermore, the probability that the response time of a BuyStocks request does not exceed 2 seconds has to be at least 0.98 , that is, the response time of at least 98 percent of all BuyStocks requests must not exceed 2 seconds. Similarly, it is required that the response time of at least 98 percent of all QueryPortfolio requests must not exceed 4 seconds. For second-class customers, at least 95 percent of BuyStocks requests must 
not exceed a response time of 2 seconds, and the mean response time and the standard deviation of QueryPortfolio requests must not exceed 1 second. The inhouse applications have relaxed performance goals as they have specified only upper bounds on mean response times and do not give restrictions on response time variability. The brokerage server has to serve requests for two different services requested by three different customer classes, leading to a multi-class workload of six different request classes. The challenge is to serve the requests in an order so that all specific performance goals are met. Most messaging products provide a priority-based service of requests where the requests of the six request classes can get different priorities leading to class-specific response times. However, it is unclear if (a) there exists a prioritization satisfying all performance goals, (b) if such a prioritization exists, how it looks like, and (c) if no such prioritization exists, which performance goals are "hardest" in the sense that they contribute most to the elimination of prioritizations and should be reviewed. To answer these questions, we have developed the HEART tool. Based on given request arrival rates and mean service times of the six request classes, the answer given by HEART for our example setting is the following: The only possible prioritization satisfying all given performance goals is to give highest priority 1 to FirstClass_BuyStocks requests, next highest priority 2 to SecondClass_BuyStocks, priority 3 both to FirstClass_QueryPortfolio and SecondClass_QueryPortfolio, priority 4 to Inhouse_BuyStocks, and lowest priority 5 to Inhouse_QueryPortfolio requests. The solution is not obvious at all as it is difficult to compare the "hardness" of the given performance goals.

\section{The HEART Tool \& Demo}

The HEART tool is a web-based system for computing request prioritizations that meet specified performance goals based on given workload parameters for a server. Figure 2 shows a screenshot of the Web interface of HEART for an example user "Frank" who models the "Brokerage Server" shown in figure 1. For each request class the administrator has to provide a (statistical) description of the expected or measured workload and the response time goals for the class. For this purpose, we have extended the GET function used to dequeue the next message from the server message queue. Our extension determines the required workload parameters for HEART automatically based on timestamp information provided by the messaging product. The performance goals are specified by entering the required mean response time, the

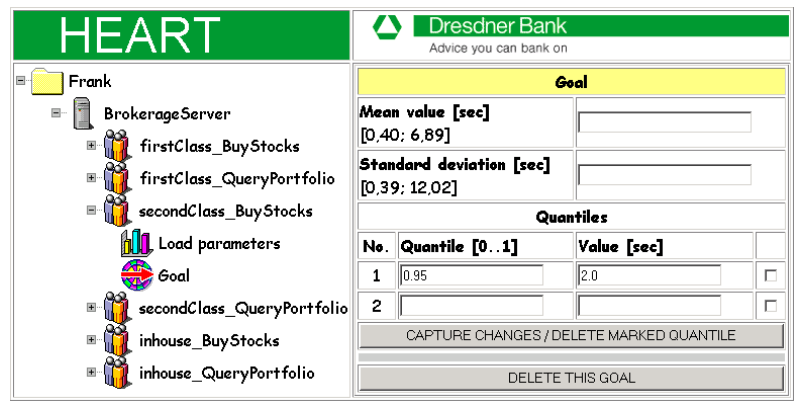

Fig. 2. HEART Screenshot 
required standard deviation, and percentiles (which have to be entered in our tool as quantile values. After the administrator (Frank) has specified all workload parameters and goals, he initiates the automatic identification of prioritizations satisfying the response time goals. Conceptually, the tool enumerates all possible priority assignments for the given classes, and assesses the performance of each such prioritizations to identify the ones that satisfy all performance goals. The number of possible prioritizations is quite large. For example, with six request classes in our case and 10 different priorities supported by MQSeries the total number of possible prioritizations is 4683 which is much too large to be identified by "trial and error" in a practical setting. The HEART tool identifies the prioritizations that satisfy all performance goals by evaluating each possible prioritization based on analytical results of stochastic queueing models and partially using simulations $[2,3]$.

The demo shows the architecture and basic principles of the HEART tool as well as an interactive elaboration on an example setting where it is shown that (a) an intuitive prioritization of requests is very difficult and in most cases impossible, and that (b) HEART is able to give the user a "feel" for the interdependencies between prioritizations and the feasibility of response time goals.

\section{References}

1. N. Bhatti, A. Bouch, A. Kuchinsky: Integrating User-Perceived Quality into Web Server Design, $9^{\text {th }}$ WWW Conference (www9.org), October 2000

2. A. Kraiss, F. Schoen, G. Weikum, U. Deppisch: Middleware-based Response Time Guarantees for e-Services (in German), $9^{\text {th }}$ German Database Conference (BTW), Oldenburg, Germany, March 2001

3. A. Kraiss, F. Schoen, G. Weikum, U. Deppisch: Towards Response Time Guarantees for e-Service Middleware, IEEE Data Engineering Bulletin 24(1), March 2001 